\title{
QUANTUM MECHANICS IN ONE BAND OF A SOLID
}

\author{
J. ZAK ${ }^{1}$ \\ Department of Physics, The University of Michigan, Ann Arbor, MI 48109, USA
}

Received 23 February 1979

Revised manuscript received 29 March 1981

The Bravais lattice operator and the quasimomentum are shown to form a complete set of conjugate operators in one band of a solid.

In elementary quantum mechanics it is customary to describe the motion of a spinless particle by means of its coordinate $\boldsymbol{r}$ and momentum $\boldsymbol{p}$ operators. In such a description any physical operator is necessarily a function of $\boldsymbol{r}$ and $\boldsymbol{p}$, and in this sense $\boldsymbol{r}$ and $\boldsymbol{p}$ form a complete set of operators [1]. In solids one often works with a one-band approximation which considerably restricts the freedom of motion of the particle. One should expect that in describing physical phenomena in one band the set of $\boldsymbol{r}$ and $\boldsymbol{p}$ will turn out to be too broad and that a more restricted set of operators should suffice for this purpose. This was actually shown by McIrvine and Overhauser [2] who derived the superlattice representation by localizing simultaneously the quasimomentum $\boldsymbol{k}$ and the Bravais lattice vector $\boldsymbol{R}$ for one energy band. In approaches to elementary dynamics of solids it is a common procedure to use one-band operators in the effective hamiltonian theories. This is shown in a comprehensive article by Blount [3] who has defined a mixed representation for dealing with band operators in the framework of Wigner's density matrix [4]. It is well known from both experiment and theory that an isolated band in a solid is a relatively stable entity under different perturbations and it seems that the physics of the problem takes place entirely in one particular band. It should be of interest to have a set of elementary operators complete with respect to one band in the same

1 Permanent address: Department of Physics, Technion, Haifa, Israel. sense as $x$ and $p$ are complete with respect to the whole physical space.

In this letter we define an operator $R_{n}$ for a Bravais lattice in a solid. The quasimomentum $k$ is shown to be conjugate to this operator and together they form a complete set of operators for one band in a solid. Any one-band operator is expressible as a function of $k$ and $R_{n}$. The eigenfunctions of $R_{n}$ are shown to be the coordinate minimal uncertainty Wannier functions for a given band. We actually show that in the framework of one band $R_{n}$ defines a quantum mechanical representation. This raises the known Wannier representation in quantum mechanics. The operators $k$ and $R_{n}$ in one band are in complete analogy with $p$ and $x$, respectively, in the whole physical space. By means of $k$ and $R_{n}$ one can develop quantum mechanics in one band of a solid.

Let us start with the calculation of the coordinate uncertainty $\Delta x$ in a general Wannier state in the $k q$ representation

$\exp \left[\mathrm{i} \phi_{n}(k)\right] \psi_{n k}(q)$,

where $\phi_{n}(k)$ is an arbitrary phase for the band $n$. In (1) the fact was used that a Wannier function in the $k q$-representation equals the corresponding Bloch function in the $x$-representation [5]. The following normalization is assumed in (1) ( $a$ is the lattice constant):

$\int\left|\psi_{n k}(q)\right|^{2} \mathrm{~d} q=a / 2 \pi$ 
The expectation values of $x$ and $x^{2}\left[x_{n n}\right.$ and $\left.\left(x^{2}\right)_{n n}\right]$ in the state (1) are

$$
\begin{aligned}
& x_{n n}=\left\langle X_{n n}(k)\right\rangle-\left\langle\partial \phi_{n}(k) / \partial k\right\rangle, \\
& \left(x^{2}\right)_{n n}=\sum_{m}\left\langle X_{n m}(k) X_{m n}(k)\right\rangle \\
& \quad-2\left\langle\left[\partial \phi_{n}(k) / \partial k\right] X_{n n}(k)\right\rangle+\left\langle\left[\partial \phi_{n}(k) / \partial k\right]^{2}\right\rangle,
\end{aligned}
$$

where

$$
X_{m n}(k)=\frac{2 \pi}{a} \int u_{m k}^{*}(q) \mathrm{i} \frac{\partial u_{n k}(q)}{\partial k} \mathrm{~d} q
$$

and the singular brackets denote an average over the band. Thus, in general,

$\left\langle A_{n}(k)\right\rangle=\frac{a}{2 \pi} \int_{-\pi / a}^{\pi / a} A_{n}(k) \mathrm{d} k$.

In (5), $u_{n k}(q)$ is the periodic part of the Bloch function. From (3) and (4) one can find the following ex. pression for the square of the coordinate uncertainty:

$$
\begin{aligned}
& (\Delta x)^{2}=\left(x^{2}\right)_{n n}-\left(x_{n n}\right)^{2}=\sum_{m}^{\prime}\left\langle X_{n m}(k) X_{m n}(k)\right\rangle \\
& +\left\langle\left[\partial \phi_{n}(k) / \partial k-X_{n n}(k)\right]^{2}\right\rangle \\
& -\left[\left\langle\partial \phi_{n}(k) / \partial k-X_{n n}(k)\right\rangle\right]^{2},
\end{aligned}
$$

where the prime in the sum means that the term with $m=n$ is excluded. Since, as can be checked, the sum in (7) is phase independent, $(\Delta x)^{2}$ achieves its minimal value when

$$
\partial \phi_{n}(k) / \partial k-X_{n n}(k)=\text { const. }
$$

This is an equation for the phase $\phi_{n}(k)$ that minimizes the coordinate uncertainty in the Wannier state (1). In order to find the physical meaning of $\phi_{n}(k)$ let us rewrite eq. (8) in the form of an eigenvalue equation (with the constant replaced by $-R_{n}^{\prime}$ ),

$$
\left[\mathrm{i} \partial / \partial k+X_{n n}(k)\right] \exp \left[\mathrm{i} \phi_{n}(k)\right]=R_{n}^{\prime} \exp \left[\mathrm{i} \phi_{n}(k)\right] \text {. }
$$

Eq. (9) has a simple physical interpretation. It can be best seen by using the Adams representation [6] for the coordinate $x$,

$x_{m n}(k)=\mathrm{i}(\partial / \partial k) \delta_{m n}+X_{m n}(k)$.
By comparing (9) with (10) we see that the operator in (9) is nothing else but the diagonal part of the coordinate $x$ in the Adams representation. We shall call this quantity $R_{n}$,

$R_{n}=\mathrm{i} \partial / \partial k+X_{n n}(k)$.

In ref. [3] it was shown that $R_{n}$ in (11) behaves like an operator under a phase transformation. What this means is that no matter how the phase is chosen in $u_{n k}(q), R_{n}$ will have the form (11) with $X_{n n}(k)$ defined in (5). With this in mind we are in a position to claim that the phase $\exp \left[i \phi_{n}(k)\right]$ that minimizes the coordinate uncertainty in the Wannier state is an eigenstate of the operator $R_{n}$ in (11).

It is easy to find the eigenvalues $R_{n}^{\prime}$ and the eigenfunctions $\Phi_{n}(k)$ of eq. (9). We have

$$
\begin{aligned}
& R_{n \nu}^{\prime}=\left\langle X_{n n}(k)\right\rangle+a \nu, \quad \nu=0, \pm 1, \pm 2, \ldots, \\
& \Phi_{n \nu}(k)=\exp \left(\mathrm{i} \int_{0}^{k}\left[X_{n n}\left(k^{\prime}\right)-R_{n \nu}^{\prime}\right] \mathrm{d} k^{\prime}\right) .
\end{aligned}
$$

The eigenvalues $R_{n \nu}^{\prime}$ in (12) cover (up to the constant $\left.\left\langle X_{n n}(k)\right\rangle\right)$ all the points of the Bravais lattice. The solutions (13), as can easily be checked, are covariant under any phase transformations and by writing them in (1) we can construct covariant minimal uncertainty Wannier functions,

$$
\begin{aligned}
& a_{n \nu}(k, q)=\exp (-\mathrm{i} \nu a k \\
& \left.\quad+\mathrm{i} \int_{0}^{k}\left[X_{n n}\left(k^{\prime}\right)-\left\langle X_{n n}(k)\right\rangle\right] \mathrm{d} k^{\prime}\right) \psi_{n k}(q) .
\end{aligned}
$$

The eigenfunctions (14) of the operator (11) form a complete set in the subspace of one band. This means that the operator $R_{n}$ defines by itself a quantum mechanical representation [1] (similar to $x$ in the full space). Since its eigenvalues assume all integer multiples of $a$ (up to the constant $\left.\left\langle X_{n n}(k)\right\rangle\right), R_{n}-\left\langle X_{n n}(k)\right\rangle$ can be called the Bravais lattice operator. An alternative way of constructing a Bravais lattice operator $R_{n}$ was given by Weinreich [7] who used for this purpose minimal uncertainty Wannier functions. A detailed discussion of the meaning of $R_{n}$ is given by Anderson [8].

Having shown that $R_{n}$ defines a quantum mechanical representation we can now look for an operator which is conjugate to it. This is not hard to find know- 
ing that in the Adams representation the wave function $B_{n}(k)$ is periodic in $k$ with the period $2 \pi / a$. This means that the translation operator $T$ which in the Adams representation is given by $T \equiv \exp (\mathrm{i} k a)$ also defines by itself a quantum mechanical representation (this should be compared with the angular coordinate [9]). In addition we have for the commutator of $R_{n}$ and $\exp (\mathrm{i} k a)$

$$
\left[R_{n}, \exp (\mathrm{i} k a)\right]=-a \exp (\mathrm{i} k a) \text {. }
$$

This is the same relation as is satisfied by angular momentum and angle. The quasimomentum, given by $T$ $\equiv \exp (\mathrm{i} k a)$ is therefore the conjugate operator to $R_{n}$. This shows that $R_{n}$ and $T$ is a pair of conjugate operators which replace $x$ and $p$ in the one-band framework. It is worthwhile to point out that the quantities $R=\mathrm{i} \partial / \partial k$ and $k$ have been intuitively used over years in many textbooks as a pair of conjugate operators. What we have shown here is that $R_{n}$ in (11) and $\exp (\mathrm{i} k a)$ can be put into an exact quantum mechanical framework (note that $\mathrm{i} \partial / \partial k$ by itself is not an operator because it is phase dependent [3]). Thus, in quantum mechanics in one band of a solid we can talk about the $R_{n}$-representation (replacing the $x$-representation) and the $k$-representation (replacing the $p$-representation). In the former, the eigenfunctions of $R_{n}$ are the complete set of the (now covariantly defined) Wannier functions (13) or (14) for one band, while the eigenfunctions of $\exp (\mathrm{i} k a)$ are the complete set of Bloch functions for one band. This completes the proof that the operators

$R_{n}=\mathrm{i} \partial / \partial k+X_{n n}(k)$ and $T \equiv \exp (\mathrm{i} k a)$

form a complete set of conjugate operators in one band of a solid.

The Wannier functions have been known for more than 40 years [10] and they are widely used in solids for defining what is called the Wannier representation. Strictly speaking, however, only in their covariant form (14) do the Wannier functions $a_{n \nu}(k, q)$ relate to a quantum mechanical representation defined by the band index $n$ and the site index $\nu$ given by the eigenvalues of the hermitian operator $R_{n}$. The additional demand that they be eigenfunctions of $R_{n}$ gives the site index $\nu$ a well-defined meaning and raises the Wannier representation to the status of a representation in quantum mechanics.

It is of interest to look at the superlattice representation [2] in view of the above developed quantum mechanics for one band of a solid. Let us choose a fixed integer $L$ and define the following operators:

$\exp (\mathrm{i} k L a), \quad \exp \left(\mathrm{i} R_{n} 2 \pi / L a\right)$.

These operators commute and they can be used for defining a $k q$-representation in the quantum mechanics of one band in the same way as the operators

$\exp (\mathrm{ipa}), \quad \exp (\mathrm{ix} 2 \pi / a)$

define the usual $k q$-representation [5]. It can be shown that the superlattice representation is defined by the operators conjugate to (17) in the same way as the Bravais and reciprocal lattices [11] are defined by the operators conjugate to (18). A detailed discussion of this problem will be given in a future publication.

The one-band quantum mechanics sheds also some light on another important problem. Consider the Stark ladder for a Bloch electron in an electric field. The latter is obtained by solving the following one-band equation in the Adams representation $[5,10]$ :

$\epsilon_{n}(k) B_{n}(k)+e E\left(\mathrm{i} \partial / \partial k+X_{n n}(k)\right) B_{n}(k)=\epsilon B_{n}(k)$.

The eigenvalues $\epsilon_{n \nu}$ and the eigenfunction $B_{n v}(k)$ of eq. (19) are

$\epsilon_{n \nu}=\left\langle\epsilon_{n}(k)\right\rangle+e E\left\langle X_{n n}(k)\right\rangle+e E a \nu$,

$B_{n \nu}(k)=\exp \left(\frac{\mathrm{i}}{e E} \int_{0}^{k}\left[\epsilon_{n}\left(k^{\prime}\right)+e E X_{n n}\left(k^{\prime}\right)-\epsilon_{n \nu}\right] \mathrm{d} k^{\prime}\right)$.

This is called the Stark ladder in solids. As can be seen the eigenvalues and eigenfunctions of $R_{n}$ [eqs. (12), (13)] are identical with the results (20) and (21) for $e E=1$ and $\epsilon_{n}(k)=0$. What this means is that the actual ladder structure is introduced by the operator $R_{n}$ itself. Eq. (19) can now be interpreted as describing a Bloch electron in the potential $e E R_{n}$ rather than in the electric field potential $e E x$. By comparing the results (20), (21) with eigenvalues and eigenfunctions [eqs. (12), (13)] of $R_{n}$ we see that the ladder structure of $R_{n}$ is very stable and is not disturbed by the introduction of the periodic hamiltonian $\epsilon_{n}(k)$.

The results of this paper can directly be generalized to a three-dimensional crystal containing a center of inversion. This can be seen by generalizing the operator $R_{n}$ in (11) to three dimensions,

$\boldsymbol{R}_{n}=\mathrm{i} \partial / \partial \boldsymbol{k}+\boldsymbol{X}_{n n}(\boldsymbol{k})$.

This is a vector and it is possible to find eigenvalues 
and eigenfunctions of $\boldsymbol{R}_{n}$ [like in eqs. (12), (13)] if its components commute. It is easy to check that the commutator of any two components $R_{n \alpha}$ and $R_{n \beta}$ of $R_{n}$ is given as follows:

$\left[R_{n \alpha}, R_{n \beta}\right]=\mathrm{i}\left[\operatorname{rot} X_{n n}(\boldsymbol{k})\right]_{\gamma}$,

with $\alpha, \beta, \gamma$ forming a right cycle. When the crystal has a center of inversion rot $\boldsymbol{X}_{n n}(\boldsymbol{k})$ is zero [3] and the components of $\boldsymbol{R}_{n}$ commute. For crystals with rot $\boldsymbol{X}_{n n}(\boldsymbol{k})=0$ eqs. (8) and (9) can be replaced by their corresponding three-dimensional versions. Solving these equations is analogous to finding the potential $\phi_{n}(\boldsymbol{k})$ for a given electric field $\boldsymbol{X}_{n n}(\boldsymbol{k})$. This is, in principle, a solvable problem and has to be considered for each particular solid separately. Having $\phi_{n}(\boldsymbol{k})$ we can construct covariant Wannier functions for three-dimensional solids in the form (13) or (14). These functions form a complete set and the operator $\boldsymbol{R}_{n}$ will therefore define a quantum mechanical representation. Again, one can then claim that $\boldsymbol{R}_{\boldsymbol{n}}$ and $\exp \left(\mathrm{i} \boldsymbol{k} \cdot \boldsymbol{a}_{j}\right)$ $\left(a_{j}\right.$ are the unit cell vectors) form a complete set of conjugate operators in one band of a solid. They replace $\boldsymbol{r}$ and $\boldsymbol{p}$ for a spinless particle.

It is interesting that in the general case where rot $\boldsymbol{X}_{n n}(\boldsymbol{k}) \neq 0$ one can still define the Bravais lattice operator (22). The minimal uncertainty of its square will lead to the usual condition on the phase of the Wannier function $[3,7]$. However, $\boldsymbol{R}_{n}$ can no longer be used in the general case for defining a quantum mechanical representation in one band by constructing eigenfunctions of $\boldsymbol{R}_{n}$. Is there another physical way of doing this ${ }^{\neq 1}$ ?

In conclusion, let us make the following remarks. First let us mention that when more than one band plays a role in the physics of the problem the relevant operators in addition to being functions of $\boldsymbol{R}_{n}$ and $\exp \left(\mathrm{i} k \cdot a_{j}\right)$ will also be matrices in the band index. One can therefore extend the above results directly to a number of bands. Having established the properties of the Bravais lattice operator one can look now at Wannier functions as being eigenfunctions of $R_{n}$

₹1 While this letter was being considered for publication two papers by the author and J.-P. Rouyet were published on the same subject [12]. In the second of these a three-dimensional Bravais lattice operator is defined. $-\left\langle X_{n n}(k)\right\rangle$ and the band index operator $B_{n}$. A physical way of choosing the latter is $B_{n}=\left\langle\epsilon_{n}(k)\right\rangle$. These two operators define the Wannier functions in the same way as the Bloch hamiltonian and the quasimomentum define the Bloch functions. The results of this letter put therefore the Wannier functions and the Bloch functions on an equal footing of being eigenfunctions of physical operators. This is of particular interest in connection with the recent study of the contrasting properties of the Bloch and Wannier functions by means of Fourier analysis [13].

As a final remark let us mention the problem of superlattices [14]. In this case the operators (17) will generate symmetric coordinates in one band in much the same way as the finite translations (18) generate symmetric coordinates in solids.

It is a pleasure to thank Dr. G. Weinreich for his interest in this work and the Physics Department at the University of Michigan for its kind hospitality.

\section{References}

[1] P.A.M. Dirac, The principles of quantum mechanics (Oxford U. P., 1958).

[2] E.C. McIrvine and A.W. Overhauser, Phys. Rev. 115 (1959) 1531.

[3] E.I. Blount, in: Solid state physics, Vol, 13, eds. F. Seitz and D. Turnbull (Academic Press, New York, 1962).

[4] E.P. Wigner, Phys. Rev. 40 (1932) 749.

[5] J. Zak, in: Solid state physics, Vol. 27, eds. H. Ehrereich, F. Seitz and D. Turnbull (Academic Press, New York, 1972).

[6] E.N. Adams, J. Chem. Phys. 21 (1953) 2013.

[7] G. Weinreich, Solids: elementary theory for advanced students (Wiley, New York, 1965).

[8] P.W. Anderson, Concepts in solids (Benjamin, New York, 1963).

[9] J. Zak, Phys. Rev. 187 (1969) 1803.

[10] G.H. Wannier, Elements of solid state theory, (Cambridge U.P. 1959).

[11] J. Zak, Phys. Rev. 12B (1975) 3023.

[12] J. Zak, Phys. Rev. B20 (1979) 2228; J.-P. Rouyet and J. Zak, Phys. Rev. B23 (1981) 561.

[13] G. Strinati, Phys. Rev. 18B (1978) 4106.

[14] J.N. Schulman and T.C. McGill, Phys. Rev. Lett. 39 (1977) 1680 . 